\title{
FAMILIA Y EVOLUCIÓN DEMOGRÁFICA EN ASTURIAS
}

\author{
Ła Baudilio Barreiro Mallón
}

Se ha probado suficientemente que existe una relación directa entre las dimensiones de las explotaciones agrarias y las del grupo familiar. En la actualidad puede decirse que es vanal la polémica entre quienes defienden que la dimensión del grupo doméstico es el factor determinante de la amplitud de las explotaciones agrarias y aquellos otros que invierten los términos y consideran que la dimensión de estas determinó en gran medida la amplitud de aquel. De hecho, los estudios elaborados a partir del Catastro de La Ensenada demuestran con toda claridad que las dimensiones de las explotaciones agropecuarias evolucionaban al ritmo de la edad de los cabezas de familia, describiendo una parábola al alza a medida que iban naciendo los hijos, se cobraban las dotes y las herencias y se realizaban adquisiciones de otros bienes raíces, y a la baja desde que el cabeza de familia alcanzaba los 45 años, aproximadamente, respondiendo a la salida del hogar de los hijos con sus correspondientes dotes. Dicho de otro modo, las explotaciones agrarias aumentaban de tamaño al mismo tiempo que se ensanchaba el hogar y se contraían cuando este se iba desprendiendo de alguno de sus miembros y, por eso mismo, disminuían tanto su capacidad laboral como sus necesidades de bienes de consumo.

No obstante, lo dicho no es sino una simplificación de la realidad, aunque con un alto grado de adecuación, toda vez que el comportamiento familiar dista mucho de ser uniforme en las diversas coyunturas, tanto a la hora de decidir las formas de transmisión hereditaria como a la de desarrollar las estrategias matrimoniales, con el consiguiente impacto en la composición y estructura de la familia. Lo que sí está demostrado es la relación entre la estructura de la familia y la del sistema agrario y, además, que esta relación se mueve siguiendo los impulsos de la coyuntura ${ }^{1}$.

\footnotetext{
Dubert García, I., Historia de la familia en Galicia durante la época moderna, 1550-1830, A Coruña, 1992; Saavedra Fernández, P., "Casa y comunidad en la Galicia interior", en Parentesco, familia y matrimonio en la Historia de Galicia, Santiago, 1989; Fernández Cortizo, C., "A una misma mesa y manteles: la familia de la Tierra de Montes en el siglo XVIII", Cuadernos de Estudios Gallegos, XXXII (1982), 98; del mismo autor "En casa y compañía: grupo doméstico y estrategias familiares en la Galicia occidental a mediados del siglo XVIII", en Parentesco, familia, y matrimonio, citado ya, y "Galicia occidental a mediados del siglo XVIII: crecimiento demográfi-
} 
Al hilo de esta última, no está de más recordar lo que ha venido a ser casi un axioma indiscutible: a un período expansivo corresponde una estructura familiar predominantemente nuclear y de tamaño reducido, debido a que las posibilidades económicas provocan una cierta euforia nupcial y el consiguiente abandono del hogar por parte de los hijos a una edad cada vez más temprana, formándose de este modo muchos nuevos hogares en los que permanecen sólo los hijos más jóvenes y muy pocos colaterales. Durante este tipo de coyuntura alcista, también las familias polinucleares y extensas que pudiese haber se veían recortadas, toda vez que sus hijos disfrutaban de la misma coyuntura por lo que, al mismo tiempo, se reducía el número de colaterales corresidentes.

A la inversa, en una coyuntura regresiva, las familias nucleares probablemente tuviesen menos hijos jóvenes en el hogar, pero no necesariamente menos hijos, porque estas generaciones tenían menos posibilidades de acceso al matrimonio y quienes las tenían, solían contraerlo a edad más tardía. Por su parte, era en esta coyuntura cuando las familias complejas y polinucleares se ampliaban, no sólo, ni tanto, a causa de los hijos como de los colaterales incapaces de conseguir una dote proporcionada a su calidad; pero también se ensanchaban rodeándose de un mayor número de criados, no tanto como resultado de la oferta de trabajo en el seno del grupo familiar, cuanto por la necesidad de supervivencia de los desposeidos y por los lazos de las solidaridades familiares que dominaban en estos medios sociales.

El problema se complica si nos adentramos en el mundo de los oficios rurales complementarios. Es un hecho comprobado que la dimensión de los hogares suele ser mayor allí en donde están presentes estos oficios, lo que no siempre es fácil de interpretar: lo es cuando tales oficios sirvieron de complemento a la actividad agrícola de gran número de familias, desbordadas, en momentos de expansión agraria, por el número de hijos en relación con el tamaño de sus explotaciones. No lo es tanto si tales oficios o actividades surgieron de forma masiva como respuesta al agotaniento de los recursos agrarios, incapaces de sostener la tendencia minifundista, sin entrar en una fase de recesión; como solución se introducen entonces determinadas industrias rurales en un medio de crisis, por lo que, teóricamente, ni debería aumentar el tamaño de la familia, como grupo doméstico, ni, por supuesto, adelantarse la edad de matrimonio de estos grupos. Sin embargo, así sucedió, generando ciertas dificultades de inter- 
pretación ${ }^{2}$, las mismas que se originan a la hora de explicar por qué determinadas áreas adoptaron estas medidas, mientras que otras echaron mano del viejo mecanismo regulador que contemplaba al grupo doméstico como a un acordeón, gobernado por un jefe de casa que lo mantenía siempre bajo su rígido control, provocando que se ampliase o se contrajese según discurriese la coyuntura, pero sin que estos movimientos supusiesen ruptura alguna, ni en la estructura económica que le servía de apoyo, ni en la estructura familiar, que, muy probablemente, era la que imponía la permanencia de los sistemas agrarios de base.

El objetivo básico que nos hemos propuesto es hacer un seguimiento de los movimientos internos del grupo doméstico en el área central del Principado y, más someramente, en las comarcas del interior occidental, observados en la larga duración, de modo que pueda comprobarse si se corresponden con las incitaciones de las diversas coyunturas. En otro momento trataremos de abordar con mayor profundidad el área occidental, en donde, en nuestra opinión, la estructura compleja y amplia del grupo familiar-doméstico no es sólo el resultado de un sistema agrario basado en las superficies amplias de carácter extensivo y, con frecuencia, de aprovechamiento vecinal, sino que tal sistema familiar sirvió para impulsar o frenar el crecimiento poblacional según lo demandase la coyuntura agraria.

El área de estudio abarca los concejos costeros o muy próximos al mar, desde Villaviciosa a Castrillón, pasando por Gijón, Carreño y Avilés, y, como representativo de la zona occidental, el extenso concejo de Cangas de Tineo, observado en el tramo final del Antiguo Régimen, desde 1787 hasta 1824. Se trata de un área con una elevada densidad de población, en su parte costera y de valle -55 habts./km. ${ }^{2}$ en Villaviciosa, 65 en Gijón, 70 en Carreño, 84 en Avilés, etc., muy por encima de los 33 del conjunto del Principado en 1787- y relativamente baja en Cangas, que con sólo 17 apenas supera la midad de la cifra media asturiana.

En el mismo censo de Floridablanca aparece una serie de realidades muy dispares entre una y otra comarcas. Las tasas de masculinidad en la zona costera central eran muy elevadas en ese momento, incluso superiores a $100, \mathrm{y}$, como consecuencia, eran muy bajas las tasas de soltería definitiva tanto femenina como masculina -6 y $2 \%$, respectivamente, en Villaviciosa, 6 y $3 \%$ en Gijón, 6 y $1 \%$ en Carreño, 7 y $4 \%$ en Avilés-, lo que se complementa con una edad de acceso de la mujer al matrimonio también por debajo de la media astuariana, situada en torno a los 24.5 años -24.8 en

2 Puede consultarse el artículo de Rey Castelao, O., "La emigración a América en la cuenca media del Ulla: un ejemplo de análisis comarcal", Revista da Comisión Galega do V Centenario, 4 (1989), 177-217. Interesa especialmente el capítulo dedicado a emigración, comportamiento familiar y mecanismos de herencia. 
Villaviciosa, 22.5 en Gijón, 23.6 en Carreño y 23.1 en Avilés-. En cambio, la estructura interna de la población de Cangas de Tineo -compuesta por 14.150 habitantesera bastante complicada y no del todo coherente: un índice de masculinidad bastante alto, 90/100, pero también unas tasas de soltería altas $-21 \%$ para las mujeres, $12 \%$ para los hombres- $y$, por el contrario, la edad femenina de acceso al matrimonio se sitúa en 22.5 años, dos puntos por debajo de la media regional, en línea con las comarcas centrales.

Los datos de la comarca costera central indican ya que nos encontramos en un área que a lo largo de la segunda mitad del XVIII estaba atravesando un espléndido momento demográfico y económico, como, de hecho, así era, toda vez que esta costa central creció durante ese período alrededor de un $47 \%$ en términos económicos y de un $33 \%$ en términos poblacionales. En cambio, el área montañosa centro-occidental no superó entonces el $1.6 \%$ de crecimiento agrario y el $5.3 \%$ de crecimiento demográfico ${ }^{3}$. Una vez sentado esto, queda una pregunta en el aire: ¿cuál era y cómo evolucionó la estructura familiar en estas comarcas?

\section{LOS PADRONES DE HIDALGUIA O DE MONEDA FORERA}

Se trata sin duda de una de las mejores fuentes posibles para hacer un seguimiento de la evolución demográfica y socio-estamental en la larga duración, siempre y cuando se manejen con cuidado. Formalmente eran unos cuadernos en los que figuraban todos y cada uno de los jefes de casa, clasificados según su estrato social, a partir de cuya clasificiación quedaban exentos o no del impuesto de moneda forera, despreciable como carga económica pero importante por su significado de impuesto directo y personal, lo que, por derivación, llevaba consigo el reconocimiento de todas las ventajas o inconvenientes propios de la condición de noble o de pechero.

Su interés social explica que a partir de mediados del XVII estos padrones registrasen no sólo a los cabezas de familia, sino también a todos los hijos varones que permanecían en el hogar y que recibían del padre la correspondiente calidad de noble o de pechero, no siendo raro encontrar hijos considerados todos ellos como hidalgos, mientras que la madre figuraba como pechera o que de entre los hijos de una misma madre, unos fuesen hidalgos y otros, en cambio, pecheros. Por otra parte, la importancia que tenía la conservación indiscutible del estatuto de hidalgo explica que también se hiciese constar a los ausentes -al menos mientras el grupo familiar mantenía vivo su recuerdo- en los correspondientes padrones, circunstancia que convierte a es-

$3 \quad$ Véase nuestro artículo "Producto agrario y evolución de la población en Asturias, siglos XVI al XIX”, Boletín del Instituto de Estudios Asturianos, 1990, 73-96. 
tos en una fuente de primera calidad e interés para conocer los ritmos migratorios a lo largo de la época moderna.

Más dudoso es su valor y utilidad para el estudio de la evolución del grupo doméstico, aunque quizá sea más correcto decir que su empleo por el historiador no resulta fácil y que por esa razón no pueden abordarse sin matizaciones:

A) No puede pretenderse, ni aquí se pretende, convertir a los Padrones en una fuente que nos lleve a descubrir las diversas estructuras de la familia en sus múltiples y diferentes aspectos y en cada momento, a pesar de lo cual tampoco es descartable este objetivo, por lo que intentaremos ponerlo de manifiesto a través del análisis de los padrones de dos localidades, Cangas de Tineo, interior y occidental, y Llanes, costera y oriental, deteniéndonos inicialmente en el primer caso ${ }^{4}$ :

\section{Cangas de Tineo}

\begin{tabular}{|c|c|c|c|c|}
\hline & 1787 & 1807 & 1815 & 1824 \\
\hline$\%$ Solitarios & 14,9 & 18,4 & 14.8 & 15.6 \\
\hline$\%$ Con un hijo & 19,5 & 17,4 & 14.8 & 13.9 \\
\hline$\%$ Con dos hijos & 13,7 & 7,6 & 15.8 & 7.8 \\
\hline$\%$ Con más de dos & 8,0 & 8,6 & 10.1 & 11.3 \\
\hline$\%$ Con hijos y otros & 26,4 & 32,6 & 29.7 & 37.4 \\
\hline$\%$ Con hermanos & 9,1 & 9,7 & 6.9 & 5.2 \\
\hline \% Con hermanos y otros & 3,4 & 2,1 & 4.9 & 3.4 \\
\hline$\%$ Con otros & 4,6 & 3,2 & 3.9 & 4.3 \\
\hline Vecinos controlados & 209 & 221 & 242 & 276 \\
\hline Habitantes & 774 & 619 & 654 & 845 \\
\hline Habitantes/hogar & 3,7 & 2,8 & 2.7 & 3.1 \\
\hline
\end{tabular}

Resumiendo la información de esta tabla, podría decirse que el conjunto de las familias extensas y polinucleares era muy significativo y que tendió a incrementarse, pasando del $43.5 \%$ en 1787 al $47 \%$ en $1807,45.4 \%$ en 1815 y $50.3 \%$ en 1824 . Simultáneamente tendió a aumentar el número de familias con más de dos hijos bajo patria potestad -del 8 al $11.3 \%$-, permaneciendo prácticamente estable el grupo de los solitarios, salvo en el momento crítico de 1807 , cuando se dispara su porcentaje; tienden a disminuir, en cambio, las familias con sólo uno o dos hijos. Dicho de otro modo: durante este período de casi cuarenta años, coyunturalmente situado entre el estancamiento

$4 \quad$ La información correspondiente a Cangas está tomada de "Linajes de Asturias", Hidalguía, 1967, y ha sido sometida a elaboración por nosotros. 
y la crisis que afectan a esta zona -Cangas no creció más de un $2 \%$ entre 1752 y 1827-, las dimensiones del grupo doméstico se ampliaron de forma clara y lo hicieron sobre la base de mantener en su seno a un número cada vez mayor de colaterales y otros parientes, quienes seguramente sufrieron las dificultades de la coyuntura a la hora de plantearse la creación de un nuevo hogar, aunque en ello también jugase una baza decisiva la "cultura familiar" que actuaba como moderadora de la coyuntura. Esa dinámica de ampliación del tamaño familiar en momentos de crisis y de contracción en los de expansión, es propia de las áreas y grupos sociales en los que predominaban las familias amplias y complejas.

Frente a esos resultados, los del área costera del concejo de Llanes, correspondientes a 1831, ofrecen una imagen completamente distinta de la estructura de la familia, aunque no de las dimensiones de esta. Veámoslo 5 :

\begin{tabular}{lrr} 
Vecinos & 4.413 \\
\hline Habitantes & 15.633 \\
\hline Habitantes/hogar & 3.5 \\
\hline Número total de hijos & 8.150 & \\
\hline Hijos/hogar & 1.8 & \\
\hline Cabezas de familia casados & 2.920 & $64.8 \%$ \\
\hline Cabezas de familia viudos & 193 & $4.7 \%$ \\
\hline Cabezas de familia viudas & 704 & $18.0 \%$ \\
\hline Cabezas de familia solteros & 196 & $4.9 \%$ \\
\hline Cabezas de familia solteras & 192 & $4.3 \%$ \\
\hline Otros & 134 & $2.8 \%$ \\
\hline
\end{tabular}

Una rápida observación al escaso número de solteros/as y de "otros miembros" de la familia en el concejo de Llanes, es suficiente para percibir la inexistencia de familias complejas, algo ya demostrado por los datos catastrales de la zona centrooriental y que, por lo tanto, no es el resultado de los criterios empleados en la realización de los padrones de hidalguía. En cualquier caso, tampoco las áreas dominadas por la familia nuclear podían eludir una significativa presencia y cohabitación de dos núcleos en la mayor parte de los hogares que no fuesen de nueva creación, como consecuencia del matrimonio del hijo que habría de heredar la casa paterna.

5 Datos procedentes de Díaz Noriega, J.A., "Padròn de Llanes, 1831", Hidalguía, en diversos números desde 1972. Elaboración propia. 
Si antes hemos expuesto el perfil demográfico de Cangas, conviene advertir aquí que Llanes, con sus 13.250 habitantes en 1787 , presentaba una relación de masculinidad de 91/100, una baja soltería definitiva en ambos sexos $-7.8 \%$ entre las mujeres, $2.8 \%$ entre los hombres- y una edad nupcial femenina similar a la del conjunto asturiano -24.3 años en el caso que nos ocupa-. De ello se deducen los fuertes contrastes en algunas variables en ambos lugares: contraste en la edad matrimonial y en las tasas de soltería y, en cambio, una relación de masculinidad muy semejante, todo ello dentro de estructuras agrarias y familiares diferentes -en lo que a estas atañe, predominio nuclear en Llanes y amplia presencia de familias extensas en el interior cangués.

Consideramos que queda demostrado que los padrones pueden, muchas veces, servir como indicadores de la estructura familiar y de su evolución, aunque no todos ni siempre responden a ese objetivo.

B) Por otra parte, tampoco todos los padrones permiten la comparación con el conjunto, sino que suelen aparecer bloques de padrones de una misma localidad elaborados con criterios diferentes, que es preciso conocer bien para poder establecer aquellas comparaciones. Así, por ejemplo, los padrones de Carreño -costa central asturiana-, de buena conservación de 1586 a 1831, se elaboraron de forma muy simple hasta 1640 , sin ofrecer otros datos que el nombre del cabeza de familia y su condiciòn social, de modo que sólo son útiles para estudiar la evolución demográfica y social de la comarca, si bien es cierto que con una alta cota de fiabilidad, como puede deducirse de la primera variable ${ }^{6}$ :

\begin{tabular}{ccr} 
Fecha & Vecinos & Indice \\
\hline 1586 & 531 & 100 \\
\hline 1596 & 542 & 102 \\
\hline 1602 & 417 & 78 \\
\hline 1607 & 433 & 81 \\
\hline 1620 & 477 & 89 \\
\hline 1631 & 537 & 101 \\
\hline 1635 & 552 & 106 \\
\hline
\end{tabular}

Sin embargo, los datos del padrón de Carreño se disparan en 1640 hasta 932 vecinos y en 1644 hasta 990, retomando la normalidad evolutiva a partir de 1650 , con 685 vecinos y 1.515 habitantes, resultado de sumar los jefes de casa, tanto varones como mujeres, más los hijos varones, únicos que de modo sistemático empiezan a figurar en los nuevos padrones. El problema de los padrones de 1640 y 1644 radica en que, por primera vez, se contó nominalmente a todos los hijos varones, pero en lugar

6 Los Padrones de Carreño y Avilés están depositados en el archivo municipal de esta última y el de Gijón en el suyo. Conviene advertir que la evolución que aquí se presenta concuerda con la del total del Principado. 
de empadronarlos como una sola unidad con sus respectivos padres, se censó a cada grupo de hermanos como unidades separadas, si bien declarando la paternidad de cada uno. Este último dato es lo que permite, por cierto, reconstruir para todo el concejo de Carreño el número de hijos de cada familia que permanecían en el domicilio paterno; se deduce a partir de él que en 1640 la estructura familiar era la siguiente: un 20 $\%$ de los hogares correspondía a matrimonios que no tenían hijos varones en su campañia, un $32 \%$ a los que tenían uno y un $35 \%$ a los de dos o más; el $9 \%$ de los hogares estaba regentado por un viudo/a con hijos y un $3 \%$ se componía de cabezas de hogar solteros o acompañados de parientes femeninos ${ }^{7}$. Estos resultados se confirman con los de 1644 y revelan, además, que la media de hijos varones por hogar era de 1.2, dato muy importante e indicativo porque permite comparar las dimensiones del componente masculino de las familias de este momento con los datos del Catastro de La Ensenada. Por otra parte, es rotunda la demostración de que partimos de una estructura familiar simple y nuclear, aunque se irá consolidando, con pequeños e interesantes matices, en el futuro.

Queda claro, pues, que los padrones reúnen una información muy importante sobre la estructura familiar, pero también que la comparación entre ellos deberá de hacerse por bloques, previamente agrupados de acuerdo con los critierios que rigieron en su elaboración.

\section{LOS RESULTADOS DEMOGRAFICOS DE LOS PADRONES:}

Es necesario someter los padrones a un breve examen de validez como fuente demográfica, no sólo para los períodos en los que escasean los datos parroquiales, operación que ya hemos realizado, sino a partir de ese momento -aproximadamente desde mediados del XVII-, por dos razones: en primer lugar, porque desde entonces los padrones descomponen los hogares y mencionan a todos los hijos varones de estos, circunstancia que permite seguir detalladamente la evolución simultánea de vecinos y de habitantes varones a lo largo de todo el período moderno; en segundo lugar, porque esa doble evolución puede someterse a la comparación con los datos parroquiales y a la interpretación de sus resultados a la luz del conjunto de variables que configuran las diversas coyunturas económico-demográficas. Evidentemente, habría que añadir que si los padrones no resistiesen el análisis demográfico, difícilmente podrían aceptarse como indicadores de la evolución del grupo doméstico.

Los resultados no son demasiado sorprendentes. Si partimos de que trabajamos con una fuente que margina a la mujer, se comprende que el número de solitarios debe figurar muy infravalorado, al igual que el de viudas con o sin hijos. Aclarado esto, los resultados concuerdan de modo aceptable con los expresados en trabajos citados en la primera nota. 
Trabajando sobre un total de 176 series de diezmales y 46 recuentos demográficos parroquiales, tiempo atrás hemos llegado a la conclusión de que la población de Asturias aumentó durante la primera mitad del XVII un $24 \%$ en los valles y un $11 \%$ en la costa; sobre tales resultados, podemos matizar ahora que en ciertas comarcas del interior montañoso pudo haberse dado un crecimiento muy importante con base en una recuperación muy elemental del espacio abandonado con motivo de la peste de fines del XVI ${ }^{8}$-de aceptar la información parroquial, el área montañosa de Lena habría crecido en torno al $38 \%$-. Tales resultados resisten muy bien la comparación con los deducidos de los padrones o censos de 1591 y 1631 , que registran un crecimiento del $36 \%$ entre ambas fechas en el conjunto de Lena, del $48 \%$ en Somiedo, del $37 \%$ en Miranda, del $36 \%$ en Quirós, etc., si bien es cierto que en algunos concejos se aprecian pérdidas claras de población. Durante la segunda mitad del XVII, el incremento poblacional fue de un $52 \%$ en los valles y de un $82 \%$ en la costa, mientras la montaña crecía muy poco y de forma diversa hasta fines de los años setenta, pero disminuía en el conjunto de esta mitad de la centuria -la disminución equivale al $50 \%$ del número de nacimientos en Lena y el $20 \%$ en Pola de Allande-. El fuerte crecimiento global del XVII se estancó en términos generales entre 1670/85 y 1740/50, período en el que el incremento registrado en los valles es de sólo un $7 \%$ y de un $14 \%$ en la costa, para recuperarse y subir un $38 \%$ y un $32 \%$ respectivamente desde mediados del XVIII hasta 1820/29; durante este mismo siglo el crecimiento de la zona de la montaña interior centro-occidental fue del $27 \%$ en la primera mitad y del $5 \%$ en la segunda 9.

La información mantiene en la penumbra todo el siglo XVI y por esta razón sólo podría ser iluminado mediante algunos censos y padrones, o a través de la información indirecta de tipo económico. Sin embargo, estos padrones aclaran con notable precisión, no sólo las dimensiones de la crisis finisecular, sino también la rápida recuperación posterior, como puede observarse en la evolución de las cifras de vecinos, expresadas en índices, de algunos puntos clave de la costa central y de la montaña:

\begin{tabular}{lrrrrrrrrrrr} 
Concejo & 1585 & 1590 & 1596 & 1602 & 1607 & 1614 & 1620 & 1632 & 1638 & 1640 & 1650 \\
\hline Villaviciosa & 100 & 96 & 113 & 67 & 62 & - & - & 71 & 89 & - & 102 \\
\hline Gijón1 $^{100}$ & 100 & 88 & 91 & 79 & 88 & - & 87 & 97 & 101 & 116 & 119 \\
\hline Gijón $^{2}$ & 100 & 96 & 107 & 91 & 125 & - & 155 & 166 & 169 & 236 & 267 \\
\hline Carreño $^{100}$ & 100 & - & 102 & 78 & 81 & - & 89 & 101 & 104 & - & 129 \\
\hline Somiedo $^{3}$ & 100 & - & - & 68 & 78 & 85 & 91 & - & - & - & - \\
\hline
\end{tabular}

1 Rural; 2 Urbano; ${ }^{3}$ Montaña

$8 \quad$ Barreiro Mallón, B., "Los montes comunales y la coyuntura socio-económica en el Occidente de Asturias en los siglos XVI-XVIII", Homenaje a J. Uría, en prensa.

$9 \quad$ Véase nuestro artículo citado en nota 3. 
En todos los casos se confirma una caída fortísima de la población antes de 1602, aunque sin duda hubiera sido de mayor magnitud si se pudiesen cuantificar los múltiples datos cualitativos anteriores a 1585, toda vez que, según todos los indicios, la crisis se desata en torno a 1570 . Además, esta crisis tiene su correspondencia en la pérdida, en ese mismo período, del $42 \%$ en el valor de los arrendamientos decimales.

Se comprueba asimismo que la recuperación es concordante en todas las zonas, pero muy diversa en su intensidad, ya que si el crecimiento fue rápido y vivo en el mundo urbano, globalmente se confirman los resultados del censo de 1631, que ofrecen un incremento poblacional entre un 18 y un $21 \%$ en el conjunto asturiano. De modo particular, el área de Villaviciosa, Gijón y Carreño, pasó de un índice 100 en 1591 a 115 en 1631 , pero otros pueblos y concejos interiores aumentaron bastante más, como ya se ha dicho.

Hasta aquí, por lo tanto, puede afirmarse que los Padrones mantienen un buen nivel de fiabilidad. Es necesario ahora comprobar si la evolución demográfica desde el momento en que cambian los criterios de su elaboración y empieza a distinguirse de forma pormenorizada a la población masculina, es similar tanto si la deducimos de los datos parroquiales como si lo hacemos a partir de los padrones: si así fuese, estos cobrarían un gran valor, no sólo como fuente demográfica, sino también como referencia para descubrir los movimientos del grupo doméstico.

De la observación de la tabla 1 del apéndice se extraen algunas evidencias: a) todas las series alcanzan su máximo a fines de la década de los ochenta del XVII o a comienzos de la siguiente; b) las que mantienen mayor resistencia a la caída y crecen con más intensidad son las de los núcleos urbanos de Gijón y Avilés; c) la crisis se hace más profunda hasta 1720 , aproximadamente, y no se consigue recuperar el nivel de la década de 1680 hasta 1737/51, apareciendo entonces aquellos dos núcleos urbanos, de neuvo, como los más precoces y activos; d) el crecimiento del XVIII resultará en conjunto muy modesto, salvo en casos excepcionales como los de Castrillón o Carreño, caracterizados por una avanzada agricultura, hasta el punto de que en algunas de sus feligresías se lograba obtener incluso tres cosechas anuales. Al margen de estas excepciones, la población de Asturias había crecido mucho en el XVII y carecía de recursos para continuar en esa línea, aún habida cuenta de las nuevas roturaciones de la segunda mitad de siglo y de las consiguientes privatizaciones de tierras comunales. Ese mismo parece ser el comportamiento de la zona de montaña, como se percibe en los datos de Somiedo: índice 100 en 1674, 109 en 1692, 101 en 1699 y 96 en 1824; la explicación reside en que la patata aún no había conseguido imponerse y tampoco la industria rural había penetrado en ese mundo, en donde sólo se añadían a los tradicio- 
nales recursos agro-pecuarios, los también tradicionales de la arriería y de la emigración.

Si se procede a contrastar aquellos resultados con los de la evolución decenal de los bautismos, parece evidente, en primer lugar, que hay una concordancia clara, en líneas generales, entre la evolución de las curvas de natalidad, que constan en la tabla número 2, y las diseñadas por los padrones -tabla número 1-. En segundo lugar, que a través de los índices de bautismos se presenta a la costa central y oriental creciendo hasta la década de 1680 , como puede apreciarse y confirmarse en detalle tanto en los datos de Gijón como en los de Avilés, Carreño, Castrillón y Villaviciosa, y sufriendo posteriormente un prolongado marasmo, sin llegar a una abierta situación de crisis, hasta 1730/50, a partir de cuyo momento se retoma de nuevo la tendencia alcista.

La costa occidental, vista a través de la evolución de la natalidad, presenta ciertos matices de interés. A la espera de poder utilizar un mayor número de padrones en investigaciones futuras, emplearemos en esta ocasión el de Valdés, que, aún siendo lacunar y de brusca interrupción en 1759 , parece indicar una tendencia positiva todavía muy a finales del XVII; lo realmente significativo es, sin embargo, el desfase entre la marcha levemente favorable de la natalidad y el estancamiento o caída de los índices de los padrones:

\begin{tabular}{ccc} 
& Padrones & Nacimientos \\
\hline 1690 & 100 & 100 \\
\hline 1722 & 91 & 125 \\
\hline 1737 & 93 & 118 \\
\hline 1759 & 94 & 136 \\
\hline
\end{tabular}

La comparación puede resultar de mayor validez si se realiza sobre el concejo de Valdés únicamente, ya que de ese modo los datos contrastados se correspoden estrictamente al mismo territorio. Como puede comprobarse ${ }^{10}$ en la tabla número 3 , el desfase que antes señalábamos es más grave visto desde esa muestra e incluso se acentúa el progresivo distanciamiento si lo observamos sólo en torno al área montañosa del centro y del occidente; por ejemplo, el interior de ese mismo concejo habría pasado del índice 100 en 1692 al 89 en 1759, según los datos del padrón y, por el contrario, del 100 al 204 según los de bautismos. Podríamos presentar muchas otras cifras contrastadas, pero todas las correspondientes a esta zona occidental, sobre todo del interior, indican que la natalidad se sitúa siempre muy por encima de los padrones de

10 Fernández Ochoa, M.A., Luarca y la Tierra de Valdés en la Edad Moderna, Tesis Doctoral inédita, Oviedo, 1990. 
vecinos. Puede advertirse, por otra parte, un lejano parecido entre el comportamiento de esta comarca y el de la vecina Galicia, en la medida en que se insinúa una tímida recuperación, prácticamente abortada, en las primeras décadas del XVIII, para romper en las últimas, mientras que la zona de montaña no consigue liberarse de los frenos que sujetan su crecimiento; pero, al margen de esto, es necesario buscar la clave interpretativa de esa doble imagen de los padrones y de la natalidad, porque en ella radica el valor de los padrones como indicadores del movimiento del grupo doméstico.

\section{LA DIMENSION DEL GRUPO DOMESTICO A TRAVES DE LOS PADRONES}

En los últimos años ha proliferado en España una amplia literatura histórica dedicada a los comportamientos familiares ${ }^{11}$, de cuyo comentario prescindiremos, en la que se aprecia la deficiencia derivada de la carencia de censos nominativos, distribuidos a lo largo del tiempo, que permitan elaborar modelos, sometidos a confrontación con la evolución económica y con la información relativa a estrategias matrimoniales y sistemas de dote y herencia, a partir de los cuales puedan completarse las lagunas interpretativas que deja el diálogo bilateral entre la población y la producción -con sus directos mecanismos correctores- y puedan, incluso, sorprendernos si en algún caso las estructuras familiares fueran por delante de hipotéticos cambios agrarios. Consideramos que los padrones pueden cubrir la carencia de los censos nominativos y sobre esta hipótesis trataremos de ver si permiten hacer un buen seguimiento de las dilataciones y contracciones del grupo doméstico, tomando siempre como referencia el Catastro de La Ensenada.

\section{A) Concejos de Avilés y Gijón:}

En 1752 el concejo avilesino tenía 1.054 vecinos y 3.898 habitantes, que se desglosan así: 795 hijos y 764 hijas bajo patria potestad; 287 criados/as; 805 varones y 249 mujeres cabezas de familia; 675 esposas de los cabezas de familia casados; 323 correspondientes a situaciones diversas. Otros datos significativos son la cifra media de habitantes por hogar, 3.6, el índice de masculinidad entre los hijos, 104/100 y, sobre todo, el número de hijos varones por hogar, 0.75 , idéntico al que se declara en los padrones; conviene tener en cuenta, además, que existe un $6 \%$ de familias con colaterales, sólo un $3.4 \%$ con ascendentes y apenas un $1 \%$ con un segundo núcleo descendente. Los resultados del censo de 1787 son muy similares -4.1 habitantes y 0.97 hijos varones por hogar y carencia total de familias complejas-, pero no debe descui-

11 A pesar de esa afirmación, la bibliografía empieza a ser ya inabarcable y los grupos de trabajo sobre el tema son numerosos y sus resultados de notable interés, aunque su general conocimiento nos exime de exponerlos. 
darse esa pequeña variación al alza en los dos primeros datos en un momento expansivo y, por el contrario, la disminución espectacular del número de hogares sin núcleo y de solitarios: todo parece indicar que una buena parte del crecimiento demográfico es absorbido, al menos en estas áreas de predominio nuclear, mediante la ampliación del grupo doméstico, aún cuando las mejores espectativas económicas deberían de reducir el número de hijos en el domicilio familiar al potenciar sus posibilidades nupciales.

Por su parte, en 1752 el concejo de Gijón sumaba 2.525 vecinos y 9.593 habitantes distribuidos de este modo: 2.302 hijos y 2.102 hijas residentes en la casa; 487 criados/as; 2.014 varones y 511 mujeres cabezas de familia; 1.789 esposas de los cabezas de familia casados y 409 individuos de diversa condición. La cifra media de habitantes por hogar era de 3.7 y 0.9 el de hijos varones, mientras el índice de masculinidad entre los hijos era 109. Los resultados son similares a los avilesinos, aunque el desarrollo de la producción y comercialización del cobre atrajo hacia Avilés a un importante número de jóvenes solteros que rebajan la cifra media de habitantes por hogar e incrementan el porcentaje, bajo en cualquier caso, de solitarios -en 1752 Avilés tenía un $11 \%$ de solteros en esas condiciones.

¿Cómo se ha llegado a una situación como la descrita? A través de la tabla número 4 del apéndice obtenemos desde 1635 una visión panorámica de la que se concluye, en primer lugar, que durante coyunturas alcistas los incrementos del índice de habitantes son siempre muy superiores a los del número de vecinos: dicho de otro modo, aunque en situaciones favorables existe una tendencia a aumentar el número de hijos que acceden al matrimonio y a rebajar la edad nupcial media -por consiguiente, también la edad en la que los hijos abandonan el hogar-, lo cierto es que en tales circunstancias la familia incrementa el número de sus componentes. Este comportamiento se constata, sobre todo, a lo largo del XVII, salvo en su tramo final, y, en menor medida, durante la segunda mitad del XVIII, toda vez que en este último período el desfase es más moderado en consonancia con un crecimiento asímismo más moderado que en el XVII. Por el contrario, en períodos o coyunturas negativos o de estancamiento global, las oscilaciones del número de vecinos y de habitantes resultan muy similares e incluso se aproximan las tendencias que antes se habían distanciado: ese es el mensaje que se deriva de los comportamientos familiares del período de fines del XVII a la década de 1740, aproximadamente.

En segundo lugar, parece deducirse que durante el siglo XVII, de tendencia muy positiva, los resultados fueron más favorables para el sector urbano o rururbano -Avilés lo es casi íntegramente-, que para el mundo rural, verdadero impulsor del crecimiento; esto puede deberse a la probada "retirada" de antiguos comerciantes y merca- 
deres hacia sus "cuarteles de invierno", es decir, hacia la realización de inversiones en tierras y ganados sin abandonar por ello sus domicilios en villas y ciudades. En cambio, tanto en la fase de contracción anterior a 1740, como durante la mejoría posterior a esa fecha, los comportamientos son muy similares en el mundo rural y en el urbano, al mismo tiempo que se separan muy poco las respectivas curvas o indicadores de vecinos y de habitantes.

La interpretación de este comportamiento familiar resulta bastante sencilla a la luz de la documentación parroquial. No puede buscarse en posibles descensos significativos de la mortalidad infantil que pudiesen conllevar la supervivencia de más hijos y su permanencia en el hogar, ya que toda la información reunida hasta ahora juega en contra de la tesis clásica que define como "moderno" al comportamiento demográfico del Norte, o al menos la contradice en uno de sus elementos, el de la mortalidad infantil: entre 1732 y 1780 la proporción de párvulos fallecidos sobre el número de nacidos fue del $42.5 \%$ en Quiloño, según se deduce del perfecto registro llevado en el Libro de Fábrica de la parroquia; en el concejo y feligresía de Laviana todavía alcanzaba el $40.8 \%$ en 1790 , mientras que en otros pueblos oscila entre el 40 y el $48.5 \%$ en concreto, en el área rural del concejo gijonés, la media es de $45 \%$ durante todo el XVIII, si bien se carece de datos para el núcleo urbano ${ }^{12}$. Dicho de otro modo, desde esta variable no pueden esperarse ahorros considerables de población que derivasen en un crecimiento vegetativo importante.

Será necesario, pues, buscar la interpretación en el ritmo de la nupcialidad, más concretamente, en la evolución de la edad nupcial femenina y, acaso como derivación, en el número de hijos por matrimonio. En términos generales, la edad nupcial de la mujer descendió de 24.3 años a 22.1 entre la primera mitad del XVIII y la segunda aunque el descenso sólo fue constante desde la década de 1750. Como consecuencia de este proceso, se incrementa levemente el número medio de hijos: de 4.2 a 6 en La Pedrera entre ambas mitades de siglo, si bien en el primer tercio del XIX vuelve a ser de 4.8; en Jove sube de 4.1 a 4.2 y a 4.4 ; en Somió oscila de 4.3 a 4.1 y a 5.7. Unicamente estos datos justifican y explican el aumento del tamaño del grupo doméstico en sus componentes masculinos, sin relación la presencia de colaterales, siempre muy minoritarios. Ampliar la comparación a pueblos limítrofes sólo sirver para confirmar los resultados anteriores: en las parroquias de Granda y La Barreda (Silvo) el número medio de hijos por matrimonio es de 3.2 en la segunda mitad del XVII, caracterizada aquí por el estancamiento, y de 4.4 en el XVIII, debido esto último al tirón producido

$12 \quad$ Viejo González, M.I., Estructura y evolución demográfico-social del Concejo de Gijón, siglos XVI-XIX, Tesis de Licenciatura inédita, Oviedo, 1986. 
en su segunda mitad, en la que baja la edad nupcial como en Gijón; por su parte, la evolución de la fecundidad legítima en la villa de Villaviciosa es de lenta pero inexorable regresión -5.4 hijos por matrimonio en el XVII, 5.2 en el XVIII y 4.6 en las décadas iniciales del XIX-, pero ese es el signo de la natalidad en general, que tal vez la explique la relación de masculinidad al nacer y al morir -113 y 98 respectivamente en el XVII, 115 y 79 en el XVIII, 110 y 79 en el XIX inicial.

En resumen, la coyuntura económica modifica, cuando menos, las dimensiones de la familia, ampliándolas a lo largo de las fases expansivas y reduciéndolas en los períodos de recesión. Esto, como se ha visto, no puede explicarse por recortes en las tasas de mortalidad infantil, sino por una mayor precocidad de los matrimonios y por el aumento del número de hijos en los buenos momentos, y por la inversión de los comportamientos en los negativos. No debe olvidarse que nos referimos a zonas dominadas casi en exclusiva por la familia de tipo nuclear, a lo que habrá de añadirse que de los contratos matrimoniales se deduce que aquí ambos contrayentes aportan su dote -en el $93 \%$ de los contratos-, sin que apenas se detecten matrimonios "a trueque", aunque sí, durante el XVII, que los padres del novio se comprometan a no mejorar a otros hijos ${ }^{13}$.

\section{B) Concejos de Castrillón y Carreño:}

Ambos vivieron una etapa espléndida durante el XVIII. El primero de ellos tenía en 1752 un total de 497 vecinos y 2.134 habitantes, siendo 4.3 el número medio de habitantes por hogar y 1.12 el de hijos varones; apenas existían criados, debido a las reducidísimas explotaciones agrarias, y eran muy pocas las personas no descendientes del núcleo matrimonial. Una muestra del $15 \%$ realizada sobre los datos catastrales de Carreño permite observar la existencia de 1.04 hijos y 0.71 hijas por hogar y que el número de componentes del grupo doméstico era de 4.2, sin embargo permite también comprobar que esta última cifra difiere mucho de unos grupos sociales a otros -7.8 en los hogares de los regidores, 4.03 en los de labradores y 3.96 en los de comerciantes, pescadores, etc. de la villa-. También en esta zona se corrobora el predominio aplastante de las familias nucleares, como nota característica del área centrooriental. Veamos su evolución a través de calas situadas en los momentos más significativos, basadas en documentación más explícita y fiable:

13 Argüelles Granda, L., Avilés vista a través de los contratos matrimoniales. Siglos XVI al XVIII, y Lanza López, R., El Concejo de Gijón a través de los contratos matrimoniales. Siglos XVI-XIX, en ambos casos se trata de Tesis de Licenciatura inéditas (Oviedo, 1986). 


\section{Castrillón}

\begin{tabular}{|c|c|c|c|c|}
\hline & $\begin{array}{l}\text { Vecinos } \\
\text { Indice }\end{array}$ & $\begin{array}{l}\text { Habit. } \\
\text { Indice }\end{array}$ & $\begin{array}{l}\text { Nacimt. } \\
\text { Indice }\end{array}$ & $\begin{array}{c}\text { Habit./hogar } \\
\text { Media }\end{array}$ \\
\hline 1680 & 100 & 100 & 100 & 1.8 \\
\hline 1759 & 116 & 129 & 140 & 2.1 \\
\hline 1787 & 165 & 196 & 201 & 2.2 \\
\hline
\end{tabular}

\section{Carreño}

\begin{tabular}{rrrrr}
1670 & 100 & 100 & - & 1.8 \\
\hline 1686 & 109 & 114 & - & 1.9 \\
\hline 1692 & 110 & 115 & - & 1.9 \\
\hline 1737 & 97 & 91 & - & 1.7 \\
\hline 1794 & 177 & 182 & - & 1.9 \\
\hline
\end{tabular}

Es muy interesante y expresivo el escalonamiento de los indicadores de Castrillón, en donde la natalidad se duplica a lo largo del XVIII como resultado de un mayor impulso durante la primera mitad de siglo, mientras que el número de habitantes se aproxima mucho a ese ritmo y, en cambio, el de vecinos se rezaga de modo significativo respecto al de los otros, en especial en la primera mitad de la centuria. El desfase entre nacimientos y habitantes no se explica por el comportamiento de la mortalidad, según consta por los indicadores de la misma, de modo que, en consecuencia, sólo queda la explicación razonable, aunque no cuantificable por otros medios, de la emigración, que arrastraría a ese pequeño margen que indican ambos datos. Por su parte, la ralentización de la marcha del número de vecinos queda justificada, de nuevo, por el ensanchamiento del grupo doméstico en una comarca que mantuvo la tendencia alcista a lo largo de todo el XVIII, aunque con ritmos diferentes.

Partiendo de la tesis del predominio de la familias nucleares, podemos aproximarnos un poco más al conocimiento de algunos aspectos de la estructura de aquéllas:

\begin{tabular}{lrrr} 
& $\mathbf{1 6 8 0}$ & $\mathbf{1 7 5 9}$ & $\mathbf{1 7 8 7}$ \\
\hline Habitantes/hogar & 1.8 & 2.1 & 2.2 \\
\hline \% Hogares jefatura femenina & 5.0 & 8.1 & 6.9 \\
\hline \% Hogares con un solo varón & 52.9 & 33.3 & 32.7 \\
\hline \% Con dos varones (un hijo) & 20.0 & 22.0 & 28.5 \\
\hline \% Con tres o más (2 o más hijos) & 22.2 & 29.8 & 31.4 \\
\hline \% Otros & $?$ & 6.8 & $?$ \\
\hline
\end{tabular}


Al margen de las reticencias insinuadas por epígrafes como el de "otros", en el que se encierran ciertas variables referentes a la complejidad de algunas familias, debe aceptarse que la tendencia de las tres muestras indica que el número medio de hijos por hogar se incrementó de forma contínua y clara sobre la base de una constante disminución de los hogares con un solo varón y de un sistemático aumento del porcentaje de los que contaban con dos o más varones e incluso con dos o más hijos. Para despejar cualquier duda sobre el ritmo y mecanismos de esta evolución, téngase en cuenta que en 1752 el número de hijos varones por hogar era de 1.06 y de 1.1 en 1787.

La realidad y tendencia del vecino concejo de Carreño es muy similar a la de Castrillón y similar es también la media evolutiva del número de hijos por hogar, lo que se confirma por medio de la comparación entre los resultados del Catastro y los de 1794 -1.04 y 1.1 hijos respectivamente-, e incluso con los de fechas anteriores y posteriores. En esos momentos, Carreño presenta unos índices muy favorables, pues la soltería definitiva no alcanza al $6.5 \%$ de las mujeres y al $1 \%$ de los varones. A su vez, la comparación entre la evolución de la población desde 1787 a 1860 con la línea de comportamiento que venimos observando, muestra una gran coherencia: la población pasó de un índice 100 a 118 y el índice de habitantes por hogar de 100 a 117, pasando la cifra real de estos últimos de 4 a 4.7 , con un eslabonamiento perfecto desde una media de 3.6 en la villa y arrabales de Candás hasta 6.6 en la feligresía de Perbera.

Tiempo atrás hemos demostrado que la variable independiente que movía el proceso demográfico como respuesta a la dinámica de la producción, era la nupcialidad, que provocaba de inmediato su seguimiento por la natalidad, pero sin que esta ni la mortalidad modificasen sus comportamientos estructurales en lo referente a intervalos y tasas habituales. Lo mismo puede verificarse a partir de las fuentes que aquí empleamos. Ahora bien, no podemos limitarnos a esa explicación, sino que es preciso poner en relación los movimientos de expansión y contracción de los grupos domésticos con las estructuras y coyunturas agrarias; en primer lugar, con las estructuras, de las que dependen las dimensiones y comportamientos familiares -si no de dependencia, se puede hablar de concordancia entre ambos-, y en segundo lugar, de la coyuntura, en la medida en que esta influye en la evolución de aquella o a la inversa.

En líneas anteriores veíamos que la zona occidental, representada por Cangas, era esencialmente un dominio de las familias extensas y complejas y que esa complejidad aumentaba a tenor de la coyuntura. Pues bien, su economía agraria, según los datos del Catastro, respondía a estas cifras: a) la superficie catastrada suponía un $20 \%$ del total y de ésta, un $66 \%$ corresponde a tierras comunales; b) la distribución de las superficies medias de las explotaciones familiares contenía 9.8 "días de bueyes" (medida equivalente a $1.250 \mathrm{~m}^{2}$ ), 4.4 de prado y pasto y 5.3 de tierra inculta, lo que arro- 
ja un total de 19.5 días de bueyes; c) finalmente, el comunal declarado era de 19.5 días de bueyes por vecino. Conviene tener presente que existía una superficie de monte comunal que se dedicaba a la siembra de cereal mediante el sistema de rozas y que era más amplia que la declarada como de labor y de propiedad privada. Por otro lado, si a esos datos añadimos el $80 \%$ del territorio de la jurisdicción que no se hace figurar en el Catastro, se llegará a la conclusión de que sobre estas grandes superficies se apoyaba el posible desarrollo del concejo y del conjunto del área de montaña, toda vez que en esas tierras se cultivaba cerca de la mitad del centeno y se criaba la mayor parte de su amplia cabaña ganadera. Al respecto de esto último, baste decir que mientras los ingresos derivados de la ganadería asturiana suponían el $37 \%$ del total agropecuario, en Cangas se elevaban al $47.2 \%$ y al $49 \%$ en Somiedo, y que mientras el número de cabezas de ganado por familia era en Asturias de 19.6 -4.1 de vacuno, 3.8 de porcino y 11.1 de otras especies-, alcanzaba las 32 en Somiedo $-9,3.2$ y 20.1 respectivamente: la superioridad ganadera de la zona de montaña resulta manifiesta y tiene su apoyo y fundamento, claro está, en los pastos comunales ${ }^{14}$. Por lo tanto, grandes espacios, centeno y ganado son los elementos que sistemáticamente coexisten con las familias extensas.

En estas tierras occidentales, con esa estructura de las explotaciones agrarias, en parte comunitarias por cuanto un sector importante se basa en el aprovechamiento de los pastos y en la parcelación temporal para su roturación controlada por la comunidad, los sistemas de transmisión hereditaria -aún sin estudiar en profundidad-, parecen caracterizarse por el predominio de la mejora amplia en tercio y remanente de quinto, y la distribución de la legítima a la muerte de los padres, aunque una parte de ella y a su costa podía percibirse anticipadamente en forma de dote; el propio hijo mejorado debía esperar a la muerte del padre para heredar, sin recibir antes otra cosa que el compromiso o seguro de su mejora. La autoridad paterna resultaba así consolidada y, en menor medida, la materna, dada la escasez de grupos domésticos de jefatura femenina.

No cabe duda de que la viabilidad de las explotaciones familiares empujaban a los jefes de casa a retrasar todo lo posible la entrega de dotes, limitándose con frecuencia a asegurar en sus contratos las legítimas post-mortem correspondientes, al mismo tiempo que tienden a disminuir las legítimas o las dotes respectivas. En una economía agraria de tipo claramente extensivo, de baja productividad por superficie y trabajo, apoyada en los comunales y en un sistema hereditario desigual, necesariamente el grupo doméstico tiende a incrementar sus efectivos por la permanencia en su seno

Barreiro Mallón, B., "Los montes comunales...", citado ya. 
de colaterales y descendientes ante la imposibilidad de subsistir sobre legítimas tan pequeñas y que en un $52 \%$ de los casos no se cobraban hasta la muerte de los padres.

Además, claro está, en estas áreas, tales factores se agravaban durante las coyunturas desfavorables, cuando más aumentaba el tamaño del grupo doméstico. Podría esperarse que en momentos de estancamiento se procurase una disminución del número de hijos por matrimonio, por ejemplo, retrasando la edad de matrimonio, pero no siempre fue así. Es cierto que muchos concejos de esta zona occidental interior presentan una edad nupcial netamente superior a los 24.5 años de la media regional 27 en Grandas, 28 en Illano, 30 en Abres, etc.--, pero en otros la realidad era muy distinta -24 en Allande, 22.8 en Cangas, 24.5 en Tineo, etc.-. En cambio, sí se observa que el número de hijos por matrimonio, aunque con leves oscilaciones, se mantiene en un nivel muy alto: en Pola de Allande, concretamente, fue de 4.4 hijos durante el período negativo de las cuatro últimas décadas del XVII, de 5.4 en la primera mitad del XVIII -período aceptablemente positivo- y de 5.1 en la segunda -período de estancamiento-. Si se observa la evolución la evolución en índices del número de nacimientos y matrimonios y las relaciones de masculinidad al nacer y al morir, se podrá completar la explicación de ese comportamiento:

\begin{tabular}{lcccc} 
Período & Matrim. & Nacimt. & Masc. nacimt. & Masc. defs. \\
\hline $1660 / 99$ & 100 & 100 & 117 & 85 \\
\hline $1700 / 49$ & 118 & 145 & 112 & 71 \\
\hline $1750 / 99$ & 128 & 151 & 108 & 88 \\
\hline
\end{tabular}

La tendencia no es modificada, pues, por la natalidad, sino por la nupcialidad y por la emigración, única válvula liberadora junto con la tradicional dedicación arriera de la zona. En efecto, en el área de Cangas el porcentaje de ausentes, mayoritariamente, en Castilla, era el $21.9 \%$ en 1787 , el 19.8 en 1815 y el 19.9 en 1824; la estabilidad de las cifras indica que se trata de un proceso profundamente arraigado en una estructura social que expulsaba a sus excedentes, o bien los mantenía en el hogar desarrollando funciones que los asemejaban a criados sin salario y sometidos al poder y control del cabeza de familia, de una familia que en 1824 se definía por su amplitud y complejidad.

Todo lo expuesto concuerda con la realidad y se basa en información documental incontestable, pero persiste la incógnita de porqué en estas áreas no se procedió a una privatización de los amplios comunales, toda vez que sumados estos a las correspondientes legítimas podrían haber servido de base a nuevos hogares, aunque para su subsistencia fuesen precisos también la arriería u otros oficios complementarios esta- 
bles o itinerantes. Cabe preguntar también porqué, a diferencia de otras zonas, no se introdujo alguna forma de industria rural de derivados de la madera, los curtidos o incluso del lino -aunque este hubiese que importarlo-, que incrementase las posibilidades de supervivencia. Nada se hizo en ese sentido e incluso resulta sorprendente la tardía y lenta aceptación de nuevos cultivos como la patata. Parece como si nada se quisiese cambiar para mantener estables unas relaciones familiares dirigidas y controladas por quien tenía en sus manos los recursos económicos, es decir, el cabeza de familia, toda vez que los perdería en el momento y en la medida en que cualquier novedad permitiese romper aquel control al posibilitar nuevos hogares y nuevas fuentes de ingresos al margen de este. En definitiva, no afirmamos que las pautas culturales fuesen insensibles a la variación de la coyuntura agraria, pero sí que eran muy resistentes y que su inercia tuvo siempre más fuerza que el empuje demográfico y económico.

La zona costera centro-oriental, caracterizada por el predominio aplastante de las familias nucleares, como se ha visto, se basaba en realidades económicas muy distintas de la montaña:

\begin{tabular}{lcrr} 
& Castrillón & Carreño & Villavic. \\
\hline \% Superficie catastrada sobre total & 72.0 & 100.0 & 54.0 \\
\hline \% Comunales sobre superficie catastrada & 61.0 & 70.0 & 60.0 \\
\hline Laborable/vecino (días de bueyes) & 10.6 & 6.1 & 7.0 \\
\hline Prado/pasto idem. & 5.0 & 7.5 & 7.2 \\
\hline Inculto idem. & 2.0 & 6.1 & 1.0 \\
\hline Total idem. & 17.6 & 19.7 & 15.2 \\
\hline Comunal declarado por vecino idem. & 44.0 & 47.0 & 23.0 \\
\hline
\end{tabular}

De la comparación con la montaña sorprende la similitud de las superficies de labor entre ambas zonas, aunque en el interior era más baja la productividad y aún era necesario mantener parte de la tierra en barbecho, como consecuencia de lo cual, la insuficiencia de las explotaciones era mucho más grave allí que en la costa. En cambio, en las comarcas costeras se dedica más tierra a prado/pasto, lo que, en principio, puede parecer contradictorio, toda vez que la cabaña ganadera es aquí mucho menos numerosa -12.2 cabezas en Castrillón, 11.5 en Villaviciosa, 10.7 en Carreño-, pero no lo es dada la amplitud de los pastos de montaña y la estrechez de los de valles y costas; así se explica que mientras el producto ganadero equivale al $47.2 \%$ del total agropecuario en Cangas, sólo supone el 27.7 en Castrillón, el $26 \%$ en Carreño y el 19 $\%$ en Villaviciosa. 
Indudablemente, a lo largo del período moderno, las comarcas costeras habían sufrido una fuerte presión demográfica, lo que obligó a avanzar sobre la tierra inculta, pero que también se convirtieron en el escenario de las grandes transformaciones agrarias, hasta ofrecer en los siglos XVII y XVIII la imagen de una agricultura muy avanzada. De esta forma se posibilitó la constitución de nuevos hogares, sobre la base de las correspondientes legítimas, y que esta zona se convirtiese en el feudo de las familias nucleares. En efecto, las investigaciones realizadas sobre los sistemas de dote y de transmisión hereditaria demuestran que eran más igualitarios que en el interior, pero también que, en coyunturas negativas, era frecuente la promesa paterna de no mejorar a otros hijos; demuestran asímismo que el valor medio de la dote, en términos reales, tendió a decrecer: en Avilés, por ejemplo, baja de 3.288 rs. en el XVII a 2.595 en el XVIII.

En ese contex to y esa dinámica, debe recordarse que la evolución de las familias de la costa y de los valles limítrofes era diferente e incluso se movía en sentido inverso a la del interior, contrayéndose en las coyunturas negativas y ampliándose en las favorables en razón esto último del incremento del número de hijos bajo patria potestad. Si asimismo recordamos que no hubo variaciones en la mortalidad ni controles de natalidad, eso significa que nos encontramos ante una nupcialidad más precoz, como ya se ha dicho, y una menor incidencia de la emigración en las etapas positivas, y con retraso nupcial y mayor incidencia de la emigración en las coyunturas difíciles.

En definitiva, si ya se sabía que las comarcas occidentales-de interior se definían por el predominio de las familias extensas y complejas sobre una agricultura de tipo extensivo e incluso comunitario y una fuerte presencia de ganado menor alimentado en los pastos comunales, los Padrones de Moneda Forera vienen a confirmar que, en estas áreas, las familias, ya de por sí extensas, aumentan de tamaño en las coyunturas negativas, cuando los patrimonios familiares, por un lado, y las inercias culturales, por otro, impiden la parcelación de las explotaciones y la introducción de novedades y obligan a los colaterales a permanecer en el hogar; el comportamiento se modifica en los períodos de bonanza económica, que permiten la fundación de nuevos hogares, de lo que se deduce, obviamente, que la clave explicativa radica en la nupcialidad. En cambio, en las comarcas costeras, muy avanzadas y densamente pobladas, en donde predominaban las familias nucleares, estas tendían a ampliarse, manteniendo su estructura, durante los períodos de crecimiento económico-demográfico y a contraerse, al menos a estabilizarse, cuando la cayuntura económica era desfavorable. 
Tabla no 1. Evolución demográfica (índices) a través de los Padrones

\begin{tabular}{|c|c|c|c|c|c|c|c|c|}
\hline Fecha & A & B & C & D & E & $\mathrm{F}$ & $\mathrm{G}$ & H \\
\hline 1650 & 100 & - & 100 & 100 & 100 & 100 & 100 & 100 \\
\hline 1656 & - & - & 104 & 120 & - & - & 106 & 106 \\
\hline 1662 & 124 & - & 109 & 138 & - & 106 & - & 119 \\
\hline 1668 & 140 & - & 121 & 152 & - & 149 & 132 & 116 \\
\hline 1674 & 149 & - & 121 & 175 & 121 & 174 & - & - \\
\hline 1680 & 135 & - & 118 & 137 & - & - & 144 & - \\
\hline 1687 & 155 & - & - & 157 & 132 & 194 & 155 & - \\
\hline 1692 & 142 & - & - & 168 & 134 & 204 & 154 & 145 \\
\hline 1699 & 140 & - & 112 & 145 & - & 200 & 149 & 144 \\
\hline 1704 & 122 & - & 110 & 157 & 132 & 217 & 149 & 139 \\
\hline 1711 & 115 & - & 111 & 161 & 115 & 188 & 120 & 132 \\
\hline 1718 & - & - & 108 & 152 & 113 & 193 & 120 & 126 \\
\hline 1722 & 113 & - & 109 & 146 & 112 & - & - & 132 \\
\hline 1730 & 115 & - & 111 & 152 & - & 189 & 125 & 137 \\
\hline 1737 & 124 & - & 119 & 167 & 117 & - & 138 & 136 \\
\hline 1744 & 141 & - & 120 & 149 & 125 & 206 & 145 & 136 \\
\hline 1751 & 140 & - & 121 & 149 & 133 & 208 & 147 & 135 \\
\hline 1759 & 157 & 100 & 130 & 157 & 145 & 219 & 163 & 137 \\
\hline 1766 & 149 & 97 & 139 & 165 & 164 & 222 & 186 & - \\
\hline 1773 & 147 & 107 & 145 & 184 & 165 & 219 & 186 & - \\
\hline 1780 & 178 & 107 & 159 & 186 & 188 & 225 & 232 & - \\
\hline 1787 & 176 & 102 & 166 & 201 & - & 241 & 244 & - \\
\hline 1794 & 228 & 86 & 168 & 186 & 214 & - & - & - \\
\hline 1801 & 230 & 100 & 178 & 197 & 200 & - & - & - \\
\hline 1815 & 204 & 91 & 157 & 195 & - & - & - & - \\
\hline 1824 & - & - & 178 & 217 & - & 211 & - & - \\
\hline 1831 & 226 & 112 & 160 & 221 & - & - & - & - \\
\hline 1838 & - & - & - & - & 202 & 284 & - & - \\
\hline
\end{tabular}

A, Concejo de Villaviciosa; B, Villa de Villaviciosa; C, Gijón rural; D, Gijón urbano; E, Carreño; F, Avilés; G, Castrillón; $H$, Valdés. 
Tabla no 2. Evolución demográfica (índices) a través de los bautismo

\begin{tabular}{lcccc} 
& $\begin{array}{c}\text { Costa } \\
\text { central }\end{array}$ & $\begin{array}{c}\text { Costa } \\
\text { centro.ortl. }\end{array}$ & $\begin{array}{c}\text { Costa } \\
\text { occidtl. }\end{array}$ & $\begin{array}{c}\text { Valles } \\
\text { prelitor. }\end{array}$ \\
\hline $1650 / 9$ & 100 & 100 & 100 & 100 \\
\hline $1660 / 9$ & 116 & 153 & 116 & 105 \\
\hline $1670 / 9$ & 116 & 186 & 167 & 116 \\
\hline $1680 / 9$ & 150 & 163 & 193 & 132 \\
\hline $1690 / 9$ & 146 & 112 & 195 & 113 \\
\hline $1700 / 9$ & 156 & 179 & 232 & 108 \\
\hline $1710 / 9$ & 130 & 165 & 230 & 139 \\
\hline $1720 / 9$ & 141 & 175 & 244 & 145 \\
\hline $1730 / 9$ & 153 & 172 & 232 & 147 \\
\hline $1740 / 9$ & 181 & 165 & 239 & 151 \\
\hline $1750 / 9$ & 196 & 220 & 267 & 164 \\
\hline $1760 / 9$ & 243 & 205 & 274 & 205 \\
\hline $1770 / 9$ & 250 & 218 & 272 & 213 \\
\hline $1780 / 9$ & 287 & 208 & 281 & 216 \\
\hline $1790 / 9$ & 296 & 186 & 293 & 229 \\
\hline $1800 / 9$ & 276 & 172 & 311 & 232 \\
\hline $1810 / 9$ & 138 & 186 & 334 & 220 \\
\hline $1820 / 9$ & - & 258 & 404 & - \\
\hline
\end{tabular}

Tabla no 3: Evolución demográfica (índices) del concejo de Valdés

\begin{tabular}{lcc} 
& Nacimientos & Padrones \\
\hline 1656 & - & 100 \\
\hline 1660 & 100 & - \\
\hline 1662 & 101 & 121 \\
\hline 1668 & 105 & 116 \\
\hline 1680 & 102 & - \\
\hline 1690 & 119 & - \\
\hline 1692 & 134 & - \\
\hline 1698 & 132 & 137 \\
\hline 1704 & 125 & 138 \\
\hline 1710 & 131 & 133 \\
\hline 1717 & 140 & 123 \\
\hline 1722 & 148 & 119 \\
\hline 1730 & 146 & 123 \\
\hline 1737 & 166 & 120 \\
\hline 1744 & 160 & 129 \\
\hline 1751 & 157 & 130 \\
\hline 1759 & 184 & 132 \\
\hline
\end{tabular}


Tabla no 4. Evolución según los Padrones (índices) de Avilés y Gijón

\begin{tabular}{|c|c|c|c|c|c|c|c|c|c|}
\hline & \multicolumn{3}{|c|}{ Avilés } & \multicolumn{3}{|c|}{ Gijón rural } & \multicolumn{3}{|c|}{ Gijón urbano } \\
\hline & Vec. & Hab. & $\mathrm{Hb} / \mathrm{vc}$. & Vec. & Hab. & $\mathrm{Hb} / \mathrm{vc}$. & Vec. & Hab. & $\mathrm{Hb} / \mathrm{vc}$. \\
\hline 1635 & 100 & 100 & 1.1 & - & - & - & - & - & - \\
\hline 1644 & 112 & 121 & 1.2 & - & - & - & 100 & 100 & 1.5 \\
\hline 1650 & - & - & - & 100 & 100 & 1.7 & 113 & 118 & 1.5 \\
\hline 1656 & - & - & - & 104 & 116 & 2.0 & 136 & 146 & 1.6 \\
\hline 1662 & 119 & 176 & 1.6 & 109 & 125 & 2.0 & 156 & 190 & 1.8 \\
\hline 1669 & 168 & 257 & 1.7 & 121 & 130 & 1.9 & 173 & 193 & 1.6 \\
\hline 1674 & - & - & - & 120 & 133 & 2.0 & 199 & 257 & 1.9 \\
\hline 1699 & - & - & - & 118 & 138 & 2.1 & 156 & 230 & 2.2 \\
\hline 1704 & 243 & 385 & 1.8 & 112 & 129 & 2.0 & 164 & 213 & 1.9 \\
\hline 1710 & 211 & 354 & 1.9 & 110 & 125 & 2.0 & 179 & 226 & 1.9 \\
\hline 1718 & 216 & 375 & 1.9 & 108 & 120 & 2.0 & 172 & 217 & 1.9 \\
\hline 1722 & - & - & - & 109 & 119 & 1.9 & 165 & 204 & 1.8 \\
\hline 1730 & 212 & 349 & 1.8 & 111 & 127 & 2.0 & 173 & 206 & 1.7 \\
\hline 1744 & 231 & 419 & 2.0 & 120 & 134 & 2.0 & 169 & 209 & 1.8 \\
\hline 1759 & 246 & 430 & 1.9 & 130 & 154 & 2.1 & 179 & 219 & 1.8 \\
\hline 1766 & 249 & 439 & 1.9 & 139 & 160 & 2.0 & 187 & 241 & 1.9 \\
\hline 1773 & 246 & 449 & 2.0 & 145 & 172 & 2.1 & 208 & 256 & 1.8 \\
\hline 1780 & 252 & 501 & 2.1 & 159 & 184 & 2.0 & 211 & 264 & 1.8 \\
\hline 1787 & - & - & - & 166 & 196 & 2.1 & 227 & 289 & 1.9 \\
\hline 1794 & - & - & - & 168 & 203 & 2.1 & 211 & 277 & 1.9 \\
\hline 1801 & - & - & - & 178 & 203 & 2.0 & 223 & 295 & 1.9 \\
\hline 1815 & - & - & - & 157 & 210 & - & 221 & 288 & 1.9 \\
\hline 1822 & - & - & - & 178 & 252 & - & 246 & 308 & 1.8 \\
\hline 1834 & - & - & - & 180 & 270 & - & 251 & 386 & 2.3 \\
\hline
\end{tabular}

